\title{
Inside the Bronchus
}

\section{Carina Rôlo Silvestre, André Nunes, Ricardo Cordeiro, Daniel Duarte, João Eusébio, Teresa Falcão, António Domingos}

Pulmonology Department, Centro Hospitalar do Oeste, Torres Vedras, Portugal

Email: carinarolosilvestre@gmail.com

How to cite this paper: Silvestre, C.R., Nunes, A., Cordeiro, R., Duarte, D., Eusébio, J., Falcão, T. and Domingos, A. (2020) Inside the Bronchus. Open Journal of Internal Medicine, 10, 239-243.

https://doi.org/10.4236/ojim.2020.102025

Received: April 30, 2020

Accepted: June 8, 2020

Published: June 11, 2020

Copyright (c) 2020 by author(s) and Scientific Research Publishing Inc. This work is licensed under the Creative Commons Attribution International License (CC BY 4.0).

http://creativecommons.org/licenses/by/4.0/

\section{(c) (i) Open Access}

\begin{abstract}
Tumor necrosis factor inhibitors contribute to a greater susceptibility to infection or reactivation of Mycobacterium tuberculosis. Endobronchial tuberculosis has non-specific symptoms, which may delay the diagnosis. We report a case of a 21-year-old woman, with Crohn's disease, medicated with adalimumab. The patient presented with a 2 -week history of fever, dry cough, pleuritic chest pain and weight loss of $2 \mathrm{~kg}$. Chest imaging showed bilateral nodular opacities, at the right pulmonary apex. Bronchoscopy revealed a tumorous lesion in the right upper bronchus. Bronchial biopsies were suggestive of chronic granulomatous inflammation. Bronchoalveolar lavage produced a positive for acid-fast bacilli smear and culture for Mycobacterium tuberculosis. We assumed the diagnosis of tuberculosis with endobronchial manifestations. Nowadays this is a rare manifestation. Empirical treatment for tuberculosis was initiated, with steroids as an adjunct therapy to prevent bronchostenosis, with clinical and radiological improvement.
\end{abstract}

\section{Keywords}

Tuberculosis, Crohn's Disease, TNF- $\alpha$ Inhibitors

\section{Introduction}

Tuberculosis is a serious public health problem worldwide, however, mirroring the notorious asymmetry between high-income, low-income and middle-income countries [1].

Endobronchial tuberculosis refers to tuberculous infection of the tracheobronchial tree [2] [3] [4]. The exact pathophysiological mechanism is not well understood, but it seems to occur by direct extension from the parenchyma involved, via infected sputum promoting the direct implantation of the infectious agent, and erosion of the lymph node to the tracheobronchial tree, via hematogenous and lymphatic pathways [5]. Endobronchial lesions are more frequent in 
the main and upper lobes [3].

It seems to affect more women between the second and third decades of life and older people, possibly related to senescence of the immune system and patients' comorbidities leading to reactivation and reinfections of the elderly [2] [3].

The clinical presentation varies according to the location and extent of the disease [2]. There are no pathognomonic signs. Usually, the presence of non-specific insidious symptoms delays the diagnosis [3] [4].

The literature describes multiple forms of endoscopic presentation of endobronchial tuberculosis: caseous, edematous-hyperaemic, fibrostenotic, ulcerated, tumorous, nonspecific bronchitis, and granular. It represents different stages of infection [2]. Early diagnosis and prompt treatment are crucial to prevent spread and complications, such as bronchial and tracheal stenosis, atelectasis, hemoptysis, and bronchiectasis [2] [3] [4].

\section{Case Report}

We present a 21-year-old female patient, physiotherapy student, non-smoker, with Crohn's disease (CD) since the age of 14 years-old, with extraintestinal manifestations, associated with arthritis (affecting the metacarpophalangeal joints, tibiotarses and knees) and medicated with azathioprine ( $75 \mathrm{mg}$ once a day), adalimumab (40 mg every 2 weeks for 2 years, in the last year $40 \mathrm{mg}$ every week), and etoricoxib (60 $\mathrm{mg}$ once a day). Before starting therapy, interferon gamma release assay and tuberculin skin tests were made and revealed to be negative.

The patient presented with a 2-week history of low-grade fever, dry cough, pleuritic chest pain and a documented weight loss of $2 \mathrm{~kg}$ in the same period.

On physical examination she was afebrile, blood pressure of $122 / 60 \mathrm{mmHg}$, a heart rate of 75 beats/minute, eupneic on room air with SpO2 97\%. She had no abnormal findings. The blood test revealed an increased C-Reactive Protein (6.7 $\mathrm{mg} / \mathrm{dL}$ ), without leukocytosis or neutrophilia. HIV serologies were negative.

Chest imaging showed bilateral irregular peribronchovascular nodular opacities of 1 to $2 \mathrm{~cm}$, confluent at the right pulmonary apex (Figure 1, Figure 2).

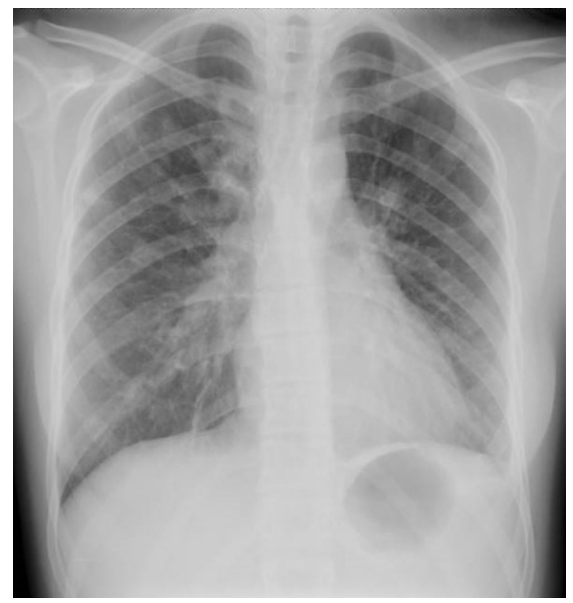

Figure 1. Chest x-ray revealing bilateral nodular opacities. 


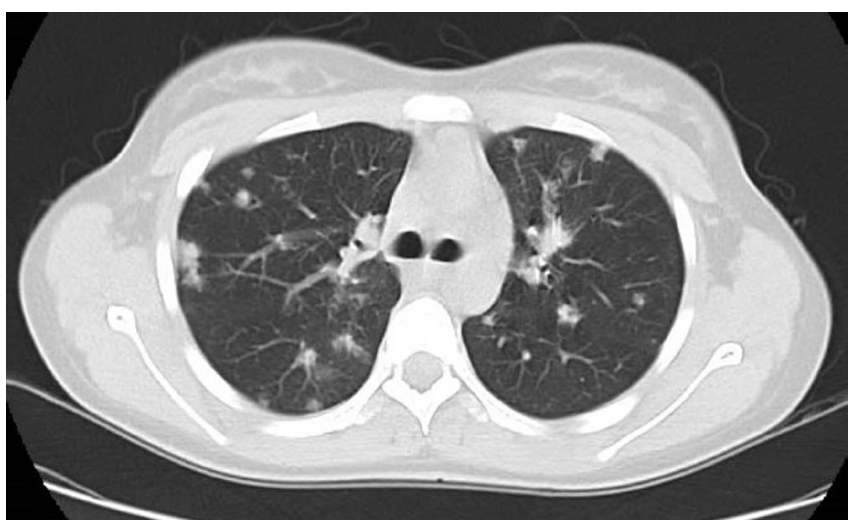

Figure 2. Chest computed tomography presenting bilateral irregular nodular opacities with a peribronchovascular distribuition, confluent at the right pulmonary apex.

Bronchoscopy showed bronchial mucosa hyperemia with an ulcerated lesion at the carina and a tumorous lesion in the right upper bronchus (Figure 3, Figure 4). In bronchial biopsies, necrosis, inflammatory infiltrate of lymphocytes, histiocytes, polymorphonuclear and eosinophils were found in high numbers, and epithelioid granulomas, highly suggestive of chronic granulomatous inflammation. Bronchoalveolar lavage gave a positive acid-fast bacilli smear, culture and Polymerase Chain Reaction for Mycobacterium tuberculosis.

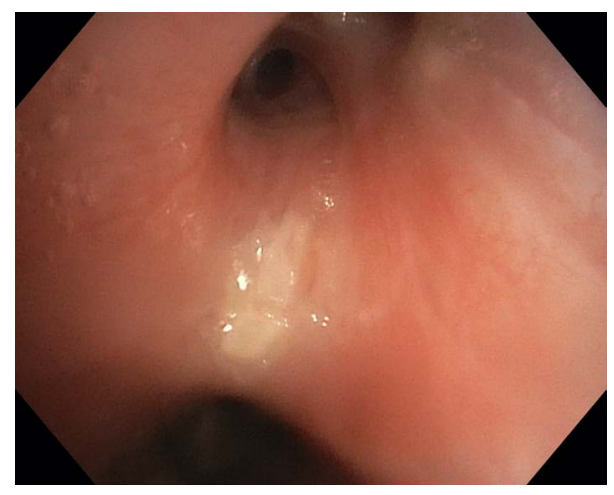

Figure 3. Bronchoscopy image revealing bronchial mucosa hyperemia with an ulcerated lesion located at the carina.

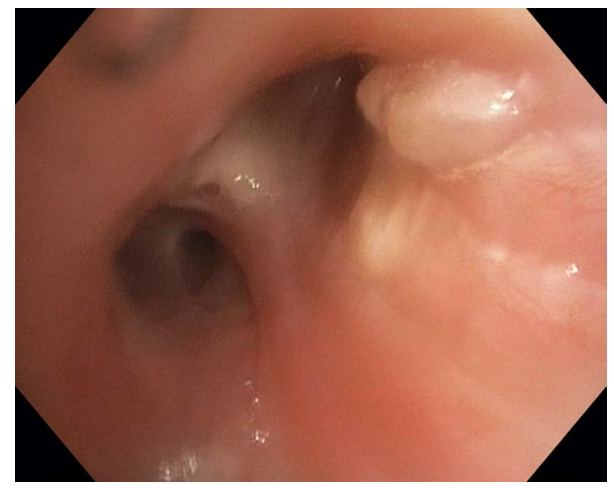

Figure 4. Bronchoscopy image showing an endobronchial tumorous lesion in the right upper lobe bronchus. 
We assumed the diagnosis of tuberculosis with endobronchial manifestations. Adalimumab was discontinued, and she started on oral prednisolone (1 $\mathrm{mg} / \mathrm{kg} /$ day) for 4 weeks followed by a slow tapering scheme of 4 more weeks, isoniazid, rifampicin, pyrazinamide and ethambutol regimen with good evolution.

\section{Discussion}

$\mathrm{CD}$ is a chronic inflammatory bowel disease that, in addition to the intestinal involvement can affect the lung. When in presence of respiratory symptoms, we must hypothesize infection, pneumotoxicity, and extraintestinal manifestations [5].

Immunomodulatory therapies like anti-tumor necrosis factor $\alpha$ (anti-TNF- $\alpha$ ) are associated with increased susceptibility to infectious diseases, due to secondary immunodeficiency. An increase in active tuberculosis such as infection or reactivation with anti-TNF- $\alpha$ treatment is an major concern, because of the macrophages reduced capacity to phagocyte and destroy mycobacteria [6] [7].

We report a case of endobronchial tuberculosis in a patient with $\mathrm{CD}$ under an anti-TNF- $\alpha$ drug, adalimumab. At present, the number of patients on immunomodulatory treatments for different diseases is growing, and they have a higher risk to develop infections. Thus special attention to tuberculosis and other infections must be considered. This case shows a nowadays rare TB manifestation.

This patient showed tuberculous infection of the tracheobronchial tree and lung. Bronchoalveolar lavage was crucial for the diagnosis of endobronchial tuberculosis.

The literature identifies that the edematous-hyperaemic, fibrostenotic, and tumorous subtypes are most often associated with stenosis within 3 months despite proper treatment [2]. Our patient had a combination of two of these subtypes that are most associated with stenosis.

Corticosteroids, were used as an attempt to prevent fibrosis, although its value is uncertain. The prognosis of endobronchial tuberculosis is variable, ranging from complete resolution to severe bronchial stenosis, the clinical course is unpredictable independently of the adequate and attempted anti-tuberculous treatment [3] [4].

Bronchoscopy follow-up is recommended to assess the possible formation of stenosis, since it can develop even with the proper anti-tuberculous and adjunct therapy with steroids.

In this case the patient refused to be submitted to a revaluation bronchoscopy, but one year after anti-tuberculous therapy she had no respiratory symptoms and no alterations on chest CT. In these situations, early diagnosis and treatment are crucial to prevent the spread of tuberculosis and complications like bronchial stenosis. A prompt diagnosis will contribute to a reduction in the morbidity and mortality associated with endobronchial tuberculosis. 


\section{Conclusions}

This case highlights the importance of a high level of suspicion for tuberculosis infection. In the developed world, the incidence of tuberculosis has decreased, being endobronchial tuberculosis a currently unusual phenomenon. Bronchofibroscopy is essential in the evaluation of endobronchial lesions.

Despite the controversy about the use of corticosteroids as a weapon in preventing stenosis, we decided to use it, considering its potential benefit, in view of the terrifying complications associated with stenosis.

\section{Ethical Aspects}

A voluntary consent form was obtained from the patient.

\section{Conflicts of Interest}

The authors declare no conflicts of interest regarding the publication of this paper.

\section{References}

[1] Furin, J., Cox, H. and Pai, M. (2019) Tuberculosis. The Lancet, 393, 1642-1656. https://doi.org/10.1016/S0140-6736(19)30308-3

[2] Siow, W.T. and Lee, P. (2017) Tracheobronchial Tuberculosis: A Clinical Review. Journal of Thoracic Disease, 9, E71-E77. https://doi.org/10.21037/jtd.2017.01.49

[3] Xue, Q., Wang, N., Xue, X. and Wang, J. (2011) Endobronchial Tuberculosis: An Overview. European Journal of Clinical Microbiology and Infectious Diseases, 30 1039-1044. https://doi.org/10.1007/s10096-011-1205-2

[4] Shahzad, T. and Irfan, M. (2016) Endobronchial Tuberculosis-A Review. Journal of Thoracic Disease, 8, 3797-3802. https://doi.org/10.21037/jtd.2016.12.73

[5] Lu, D.G., Ji, X.Q., Liu, X., Li, H.J. and Zhang, C.Q. (2014) Pulmonary Manifestations of Crohn's Disease. World Journal of Gastroenterology, 20, 133-141. https://doi.org/10.3748/wig.v20.i1.133

[6] Bongartz, T., Sutton, A.J., Sweeting, M.J., Buchan, I., Matteson, E.L. and Montori, V. (2006) Anti-TNF Antibody Therapy in Rheumatoid Arthritis and the Risk of Serious Infections and Malignancies: Systematic Review and Meta-Analysis of Rare Harmful Effects in Randomized Controlled Trials. Journal of the American Medical Association, 295, 2275-2285. https://doi.org/10.1001/jama.295.19.2275

[7] Gupta, A., Street, A.C. and Macrae, F.A. (2008) Tumour Necrosis Factor $\alpha$ Inhibitors: Screening for Tuberculosis Infection in Inflammatory Bowel Disease. Medical Journal of Australia, 188, 168-170. https://doi.org/10.5694/j.1326-5377.2008.tb01565.x 